Economía, Sociedad y Territorio, vol. vIII, núm. 26, 2008, 549-560

\title{
Neoinstitucionalismo en ciencia política
}

\author{
Shepsle, Kenneth A. y Mark S. Bonchek (2005), \\ LAS FÓRMULAS DE LA POLÍTICA. INSTITUCIONES, RACIONALIDAD \\ Y COMPORTAMIENTO, CIDE-Taurus-Santillana, MÉXico, \\ 469 PP., ISBN 968-19-1365-5
}

\section{El neoinstitucionalismo como marco analítico}

El neoinstitucionalismo es una corriente de pensamiento que se ha posicionado como uno de los paradigmas hegemónicos en las ciencias sociales. Retoma los postulados de la escuela neoclásica de la economía política, introduce una reflexión relevante acerca de los costes de transacción (North, 1990), se sustenta en el individualismo metodológico inherente a la teoría de la acción racional y utiliza como técnicas de análisis a la teoría de juegos (Elster, 1990). Esto con la ventaja de que no se idealizan, como señala José Ayala (1996), las estructuras de los derechos de propiedad ni las elecciones a favor del bien común.

Dicha corriente propone como agenda de investigación el estudio de las instituciones definidas por North como "las reglas del juego en una sociedad o, más formalmente, son las limitaciones ideadas por el hombre que dan forma a la interacción humana. Por consiguiente, estructuran incentivos en el intercambio humano" (1993: 13). La finalidad del estudio de esas reglas es explicar cómo ocurre la cooperación y por qué la cooperación no ha ocurrido en otros lugares.

Kenneth A. Shepsle y Mark S. Bonchek ofrecen un texto que desarrolla el enfoque neoinstitucionalista para el análisis de la política. En ese sentido, el rol de las instituciones políticas es dar las reglas de juego básicas como fundamento del ordenamiento de la sociedad y el marco para que funcione el sistema social. Este libro es resultado de la experiencia docente de los autores en la Escuela de Gobierno John F. Kennedy, de la Universidad de Harvard, y el objetivo enunciado por ellos mismos, que de suyo es atractivo, es "proporcionar algunas herramientas que permitan al lector llevar a cabo sus propios análisis de los acontecimientos políticos que afectan su vida” (p. 17). 
El resultado de dicha intención es que los autores ponen al alcance de los estudiantes de cualquier disciplina -tanto de licenciatura como de posgrado- de las ciencias sociales interesados en el análisis de la política, un buen material de estudio en el que tratan de manera clara los postulados teoréticos de la acción racional y del análisis de juegos, al tiempo que presentan ejemplos claros, desde la experiencia estadounidense, que permiten una mejor comprensión de las propuestas que se argumentan a lo largo del texto.

Las fórmulas de la política ofrece una interesante forma de entender uno de los problemas más intensos de la política contemporánea: la toma de decisiones. El libro fundamenta la necesidad que tiene el analista político de explicar por qué se toman decisiones y, en el caso de las organizaciones estatales, cómo ese proceso es el mecanismo que hace funcionar el sistema político y, consecuencia de ello, la generación de la acción pública.

La pregunta de investigación fundamental para quien estudia el comportamiento político, en esta vena analítica, es: ¿a través de qué mecanismos la acción, individual y colectiva, se transforma en resultados? No está de más reiterar que la inquietud fundamental de los autores es, entonces, aportar herramientas para explicar cómo ocurre la eficiencia y eficacia políticas.

\section{El contenido de la obra}

El libro se compone de 17 capítulos agrupados en cuatro partes. A continuación retomo algunos de los aspectos más sugerentes con la finalidad de motivar la lectura de este material.

La primera parte consta de dos capítulos. El primero ofrece el posicionamiento epistemológico y teórico de los autores quienes señalan que la finalidad más elevada de la ciencia es ofrecer explicaciones. Aclaran que la ciencia política, aunque no es una ciencia exacta, es una ciencia analítica que busca explicaciones y, por tanto, se aproxima al modelo de las ciencias físicas y naturales. En el segundo capítulo los autores desarrollan el enfoque toral del libro: la decisión racional. Aquí, la racionalidad se entiende como una decisión que se ajusta al modelo weberiano de la acción racional, es decir, con adecuación medios-fines. El esquema lógico a través del cual se deben identificar, pronosticar y explicar las decisiones se sustenta en la adecuación medios-fines y en el óptimo paretiano, es decir, las decisiones que toman los individuos son las que conducen a la utilidad esperada más alta. 
En la segunda parte del libro se abordan las condicionantes del proceso de toma de decisión grupal y los métodos de votación, y los autores retoman la teoría de la acción colectiva formulada por Olson como su marco analítico general.

En el capítulo tercero se señala que, salvo en casos de medidas autoritarias, en los grupos existe el problema de la diversidad de preferencias y el mecanismo más simple para solucionar ese problema es la regla de la mayoría. Sin embargo, apuntan que la regla no siempre soluciona el problema y, por ello, los individuos desarrollan comportamientos estratégicos.

El siguiente acápite profundiza en los sofisticados problemas de la decisión en grupo. Abundan acerca de la paradoja de Condorcet, es decir, individuos racionales que toman decisiones colectivas irracionales. Evitar esos resultados implica, como señala el teorema de Arrow, el equilibrio entre racionalidad y concentración de poder. La solución a la paradoja son los mecanismos institucionales que restringen la participación y la concentración de poder, tanto del agente como del principal, y refuerzan con ello la interdependencia de los individuos.

Los autores profundizan en complejidad en el quinto capítulo. Muestran cómo se analiza el proceso de toma de decisiones en una realidad multidimensional, esto es, diversos grupos que deben tomar decisiones conjuntas. Desarrollan un modelo geométrico que llaman "modelo espacial de la regla de mayoría”. Éste es una representación gráfica que -a través del teorema de Black del votante medio y el modelo Downs- sirve a Shepsle y Bonchek para explicar porqué la política de mayorías es centrípeta. El postulado es simple: más allá de divergencias por principios ideológicos entre los diversos grupos, ocurre el desplazamiento hacia el punto ideal del votante medio; aunque la convergencia o aproximación con el punto ideal no siempre es completa. En consecuencia, señalan los autores, "los convenios institucionales -los métodos políticos para las negociaciones- son profundamente importantes para los resultados que surgen del proceso político" (p. 133). Es decir, Shepsle y Bonchek reiteran que los mecanismos institucionales son el factor explicativo para comprender los acuerdos políticos.

En el sexto capítulo se trata el comportamiento estratégico. Sin asomo a alguna vertiente normativa de la filosofía política (el deber ser), se concentran en la descripción del comportamiento. Retoman el teorema de Gibbard-Satterhwaite para señalar que siempre existen incentivos para que al menos un individuo reve- 
le estratégicamente sus preferencias; es decir, que ningún método de decisión en grupo es inmune a la manipulación.

En el siguiente capítulo los autores abordan los métodos de votación y los sistemas electorales. Parten de un postulado interesante: "es casi imposible tomar decisiones en grupo que sean imparciales y coherentes” (p. 165). El énfasis está en el carácter situacional de las instituciones elegidas y demuestran, a través de casos ilustrativos, que la voluntad colectiva es algo que cambia no sólo a partir de las opciones disponibles y la determinación de la agenda. La decisión tomada está determinada por la información que aporte cada uno de los participantes, cuando expresan sus preferencias -sincera o estratégicamente- y los métodos de votación. Se introducen entonces en el análisis de los sistemas de votación por mayoría relativa y de representación proporcional.

La tercera parte del libro está compuesta por tres capítulos donde se aborda el problema del orden social. Este es el campo de análisis de los grupos intermedios que deben tomar decisiones públicas y, para evitar caer en el análisis normativo, plantean las complicaciones, paradojas y dificultades que los individuos tienen que sortear con la finalidad de lograr la coordinación social.

En el octavo capítulo, titulado "La cooperación", a partir de la teoría del Estado de Hobbes, los autores explican el por qué se constituye la sociedad civil y ese ente destinado a las actividades de protección, vigilancia y castigo a quienes infringen las reglas, el Estado, como marco en el que ocurre la cooperación. Usan el famoso dilema del prisionero como caso ilustrativo de una sociedad hipotética compuesta por dos individuos en la cual la cooperación es de suyo un asunto complejo.

El siguiente capítulo aborda el tópico de la acción colectiva desde la perspectiva de los grupos de interés. Para ello retoman la teoría de la lógica de la acción colectiva desarrollada por Olson. Para dicho autor, los individuos (racionales y maximizadores) no se organizan espontáneamente para alcanzar intereses individuales o de grupo, por el contrario, requieren de la coerción o de incentivos muy poderosos. Esto porque en el contexto de la acción colectiva ocurre el fenómeno del free rider, es decir, del gorrón o polizonte, que es el mejor incentivo para la no-cooperación.

La cuarta parte del libro está compuesta por seis capítulos donde Shepsle y Bonchek abordan la conveniencia institucional de las organizaciones gubernamentales como depositarias de autoridad y recursos para solucionar los problemas de coordinación colectiva. Aquí la dificultad central es la relación principal- 
agente. Aplican el modelo de la acción racional a las principales instituciones políticas: el líder, la legislatura, los tribunales y la democracia parlamentaria.

El capítulo decimoprimero aborda, precisamente, las características de las instituciones políticas, en particular las de gobierno, y por las cuales se les valora. 1) División del trabajo y procedimiento regular, es decir, que el resultado de que los actores se reúnan regularmente es el establecimiento de procedimientos de actuación, jerarquía y orden. 2) Especialización del trabajo: mecanismo a través del cual la organización utiliza el capital humano de sus diferentes miembros a partir de sus intereses y talentos para posibilitar la eficacia organizacional. 3) Jurisdicción: conjunto especializado de actividades de la organización. 4) Delegación y supervisión de las actividades como resultado de las necesidades de control sobre los respectivos dominios de la organización. Estas cuatro características, señalan los autores, son el común denominador que permite analizar y comprender las instituciones. Asimismo, señalan que las instituciones cambian a través de esos dos mecanismos fundamentales: la división y la especialización jurisdiccionales.

Shepsle y Bonchek se adentran en el análisis de las instituciones políticas específicas a partir del capítulo decimosegundo. En éste se atiende el tópico de las legislaturas en el que aplican el análisis de la regla de mayoría y las condicionantes de la coordinación. Sin renunciar a la idea que la legislatura -y en particular la estadounidense- es el caso ejemplar de los mercados políticos, al parecer los autores se suman al modelo de Becker (1983). Sin embargo, señalan que el proceso de toma de decisiones de actores e instituciones no es sólo resultado de la acción instrumental. Acotan el modelo propuesto por Becker a través de las cuatro funciones señaladas y apuntan que la estructura y los procedimientos de la institución política (votantes, legisladores, legislaturas, reglas de decisión, influencia e intereses) son mucho más importantes para comprender el comportamiento político.

En el siguiente acápite se abordan las relaciones entre quienes formulan las normas (legislaturas) y quienes las ejecutan (burócratas). Señalan que para analizar adecuadamente el comportamiento burocrático, se debe aplicar el modelo de la decisión racional e insertarlo en la función que cumple en el contexto del sistema político general. Utilizan tres variantes analíticas del problema principal-agente. Primero, el modelo de Niskanen según el cual las burocracias aprovechan activamente su posición 
frente a políticos pasivos; lo que resulta en aparatos burocráticos demasiado grandes, presupuestos altos y un rendimiento social inferior. Segundo, el modelo Miller-Moe que refuta el supuesto de políticos pasivos y argumenta que éstos pactan con las burocracias lo que provoca el resultado identificado por Niskanen. Tercero, el modelo McNollgast que invierte la línea de influencia marcada por Niskanen, es decir, la clase política crea burocracias y les remunera con facultades y recursos. La conclusión que se extrae de estos modelos es sencilla: las instituciones tienen costes de transacción que pueden resultar, en algunos casos, altos.

En el decimocuarto capítulo los autores aplican el modelo de la decisión racional al análisis del liderazgo. Consideran al líder como un agente y señalan que debemos observar más que las aptitudes de líder, sus intenciones. Así, en el papel de liderazgo "los roles se invierten: el amo es el servidor y el servidor, el amo" (p. 367). Es decir, el líder (agente) depende de sus seguidores (principal). Los comportamientos de los líderes están encaminados a determinar la orden del día, a actuar como emprendedores y a acumular prestigio político a favor de sus seguidores. El liderazgo, en tanto institución, es un mecanismo para solucionar los problemas de coordinación de la acción colectiva.

En el siguiente capítulo plantean la pregunta de si despojar a los jueces de los incentivos individuales los aleja del modelo de la decisión racional. Ilustran cómo se aplicaría la acción racional a los jueces señalando que, al igual que cualquier político, desean ganar influencia y prestigio en las organizaciones donde se desempeñan: los tribunales. Señalan que aunque no se esté de acuerdo con esta posición, la institución es fundamental para la elaboración de normas, ya que en el sistema social cumple la función de determinar la observancia de la ley.

El último capítulo que compone esta sección analiza la democracia parlamentaria, un caso fuera de su modelo paradigmático. Según el análisis de Shepsle y Bonchek, en el que siguen el problema del principal-agente, "los ejes de ese arreglo son la supremacía del parlamento y la responsabilidad del ejecutivo político -el gabinete, gobierno o administración- ante él” (p. 413).

Finalmente, el decimoséptimo capítulo presenta un apretado resumen de la obra. Resaltan tres lecciones que, a esas alturas, debió aprehender el lector. 1) La metodología de análisis propuesta es portátil, puede utilizarse para analizar cualquier situación política. 2) El modelo se aplica a la gran política, la que se escribe comúnmente con P mayúscula, como a la pequeña politi- 
ca, la de la vida cotidiana que se escribe con minúscula. 3) El modelo se aplica a cualquier marco institucional.

\section{Las consecuencias extraídas del libro}

Los autores, con muy poca modestia intelectual, esperan no sólo convencer al lector de las bondades del modelo propuesto, sino que "lleguen a admirarlo" (p. 53). Asimismo, en el capítulo final señalan que si el lector asumió "las lecciones" del libro, la empresa de escribirlo valió la pena; pero si no lo logró, recomiendan: "volver al capítulo 1" (p. 439).

Debemos reconocer, a su favor, que los argumentos esgrimidos resultan convincentes y el planteamiento general del libro está expuesto de manera lógica. Sin embargo, para seguir a los autores se debe tener un panorama claro de las implicaciones epistemológicas y teóricas del uso de ese instrumental analítico. Aunque cada cual sacará sus propias conclusiones y podrá considerar si es capaz de asumir en todas sus consecuencias el consejo adelantado por los autores, aquí expongo algunos de esos posicionamientos sobre los que, desde mi punto de vista, vale la pena hacer una reflexión más profunda.

De la primera parte del libro se puede desprender, sin muchas dificultades, que los autores se ubican en el positivismo crítico y consideran a la acción social, en síntesis, como el resultado de la maximización de beneficios.

En la segunda parte se puede colegir que los autores argumentan a favor de que, en el contexto de incertidumbres y comportamientos estratégicos, los procedimientos institucionales para determinar cómo se llega a la decisión son de capital importancia. El engaño, el falseamiento y la manipulación se consideran, en consecuencia, una forma sutil y matizada o extendida de racionalidad. Con ello cuestionan la existencia de algo aproximado al interés público o la voluntad general. En suma, se sugiere una reconsideración cuidadosa sobre dos de las instituciones más significativas sobre las que reflexiona la filosofía política: la justicia y la democracia.

La conclusión de la tercera parte del libro se puede resumir en que el mecanismo para entender la conducta cooperativa es la reiteración de las interacciones sociales. Es decir, los sistemas de valores interiorizados no son eficaces para explicar la coinciden-

cia de intereses entre los individuos. Sin embargo, en el caso de 
los grupos y las decisiones colectivas, la coordinación social se logra mejor a través de terceros ejecutores.

En la cuarta sección los autores dejan entrever, por tanto, una teoría voluntarista de la acción. Las instituciones son el producto de sus miembros; es decir, las organizaciones elaboran procedimientos y estructuras específicas a partir de la voluntad de los participantes; una voluntad que surge de la necesidad del individuo -en los términos de referencia teórico-abstractos de la decisión racional- para alcanzar sus intereses.

Los autores reconocen que las instituciones políticas tienen rendimientos sociales decrecientes, es decir, son costosas. No obstante, son importantes porque tienen la capacidad de emplear una amplia batería de políticas, regulaciones, reglamentaciones e impuestos para garantizar la coordinación de la acción social. Aunque existe una amplia variedad de soluciones en la experiencia humana, los autores señalan que es una institución política, el Estado, la que puede actuar como una tercera fuerza -más o menos autónoma de los grupos-, la que proporciona los incentivos u obliga coercitivamente a cooperar.

\section{Cinco observaciones sobre la propuesta}

1) Shepsle y Bonchek parten de modelos formales de análisis, por tanto, la lectura de Las fórmulas de la política no compensará los esfuerzos a aquellos lectores ansiosos de encontrar directrices normativas para la vida política. Sin embargo, cabe señalar que aunque explícitamente argumentan que se sustentan en una visión positiva de las instituciones -y no en una normativa-, en el fondo el análisis escamotea o soslaya hacer explícita $s u$ visión normativa: el pacto social.

Esa posición la podemos comprender bien a través de Bobbio (1994) quien argumenta -respecto de la sociedad occidental en un marco de instituciones democráticas- que es el pacto de no agresión de cada cual con todos los demás y la obligación de obedecer las decisiones colectivas; es decir, las reglas del juego preestablecidas de común acuerdo, el modelo normativo que le da sustento. La principal de esas reglas es la que permite resolver los conflictos sin recurrir a la violencia recíproca.

2) Shepsle y Bonchek, dando por sentada esta visión normativa, despliegan el instrumental para el análisis positivo sobre cómo los actores solucionan problemas de coordinación de la acción, de los conflictos, sin recurrir a la violencia. En ese marco, desde 
el individualismo metodológico y la acción racional, encontramos a "personas egoístas, amorales e individualistas" y por tanto no es "el mejor punto de partida para una reflexión ética" (Paramio, 2005: 31).

3) Cabe señalar que el modelo propuesto presenta problemas con el manejo del tiempo. Los autores se contentan con vagas referencias que no terminan por explicar cómo el analista de la política debe explicar esta dimensión. Convencido como estoy de que son los procesos históricos y los condicionantes culturales, en un sentido más antropológico, factores importantes para explicar el proceso político; la particular combinación de las perspectivas estructural-funcionalista y evolucionista de las que echan mano los autores para hablar de que las instituciones "avanzan con la corriente" (p. 300), me parece una solución insatisfactoria.

4) Aunque al final tenga que parafrasear al genio de la física luego de su enjuiciamiento por la Santa Inquisición, deseo subrayar la comodidad que supone organizar una demostración del comportamiento político si se dispone de un soporte teórico que permita ordenar y dar sentido a los datos positivos. Ese es, precisamente, el papel que los autores pretenden dar al entramado teórico neoinstitucionalista.

El problema es que se trata de la construcción abstracta de una asociación de individuos que extienden su acción racional maximizadora de ganancias a todos los ámbitos de su vida cotidiana. Ese individuo tendría que vivir con un alter ego superconsciente cuyas consecuencias un psicólogo podría enunciar mejor. En suma, pues, la única realidad positiva de ese individuo se encuentra en las hipótesis de los cientistas que están convencidos de la realidad superior de la vida institucional.

5) En ese sentido, resulta evidente que Shepsle y Bonchek sobreestiman el modelo analítico propuesto cuando afirman que tiene poder predictivo. Otros teóricos de la acción racional, como Elster (1990 y 2005) o North (1995), son más mesurados y afirman categóricamente que las teorías de la acción racional y la de juegos sirven para encontrar explicaciones a posteriori de la acción. Los mecanismos institucionales identificados como leyes científicas es la enunciación probabilística de ciertas regularidades del comportamiento que, en el caso de nuestros autores, convierten en una probabilidad lógica. 


\section{Coda}

Sin restar el valor de este material de estudio, no puedo dejar de señalar que en una segunda edición sería conveniente realizar una cuidadosa revisión de la forma. Además de tropezar con los errores ortográficos, en la traducción se utilizan términos que se alejan de la convención aceptada. El más evidente es el concepto principal-agente (convención) que se asienta en el libro como mandante-agente.

Asimismo, en la última parte del libro se utiliza un estilo que provoca la confusión entre organización e institución. Organización es el espacio en el que los individuos -racionales y maximizadores- deciden configurar y dar vida concreta a las normas y valores; mientras que la institución son esos principios abstractos (normas y valores) cuyo papel es estructurar la interacción social.

Resulta muy positivo que la edición en español circulara en librerías en la segunda mitad del año 2005, apenas un año después del original en inglés. Con esto resalto y aplaudo el esfuerzo editorial de Taurus y el CIDE por poner al alcance del público hispanoparlante un texto que sin duda será considerado, en breve, un clásico de la ciencia política.

Para terminar invito a una lectura atenta de este libro y, luego de las observaciones críticas vertidas, enuncio tal como lo hiciera Galileo: "Y sin embargo, sirve".

Recibido: 19 de abril de 2007. Aceptado: 6 de julio de 2007.

Luis RodríGuez CASTILlO Universidad Nacional Autónoma de México (UNAM) correo-e: 1rodriguez@cmq.edu.mx

\section{Bibliografía}

Ayala-Espino, José (1996), Mercado, elección pública e instituciones. Una revisión de las teorías modernas del Estado, Porrúa-unAm, México.

Becker, Gary S. (1983), "A Theory of Competition among Pressure Groups for Political Influence”, Quarterly Journal of Economics, 98, MIT Press, UsA, pp. 371-400. 
Bobbio, Norberto (1994), Estudios de historia de la filosofía: de Hobbes a Gramsci, Fondo de Cultura Económica, México.

Elster, Jon (1990), Tuercas y tornillos; una introducción a los conceptos básicos de las ciencias sociales, Gedisa, Barcelona.

Elster, Jon (2005) "A favor de los mecanismos", Sociológica, año 20, 57, Universidad Autónoma Metropolitana-Azcapotzalco, México, pp. 239-274.

North, Douglas C. (1990), "A Transaction Cost Theory of Politics", in Journal of Theoretical Politics, 2(4), pp. 355-367.

North, Douglas C. (1993), Instituciones, cambio institucional y desempeño económico, México, Fondo de Cultura Económica.

North, Douglas C. (1995), "Five Propositions about Institutional Change", en J. Knight e I. Sened (eds.), Explaining Social Institutions, University of Michigan Press, Michigan, pp. 15-26.

Paramio, Ludolfo (2005), “Teorías de la decisión racional y de la acción colectiva", Sociológica, año 20, 57, Universidad Autónoma Metropolitana, México, pp. 13-34.

Luis Rodríguez Castillo. Es investigador asociado "C" de tiempo completo adscrito al área de problemas contemporáneos de Centroamérica y Chiapas en el Programa de Investigaciones Multidisciplinarias sobre Mesoamérica y el Sureste del Instituto de Investigaciones Antropológicas, Universidad Nacional Autónoma de México (Proimmse-IIA-UNAM). Actualmente desarrolla el proyecto de investigación Procesos políticos, gestión local y pluralismo cultural en Chiapas, México (1970-2005). Una perspectiva antropológica de las políticas públicas, como parte de sus estudios de doctorado en ciencias sociales en El Colegio Mexiquense, A. C. Entre sus últimas publicaciones se encuentra la edición bilingüe tzeltal-español del libro Maravilla Tenejapa, Sk'op Ya'yejal JtejkLum, Conaculta, SEPI, CELALI, Gobierno del estado de Chiapas, Tuxtla Gutiérrez, 2006. Artículos en revistas indexadas como "Notas socioantropológicas sobre el Estado", Perfiles Latinoamericanos, 28, revista de la Facultad Latinoamericana de 
Ciencias Sociales (flacso), México, pp. 185-212 (2006); "Etnicidad y ciudadanía en los 'márgenes del conflicto'. La lucha por la territorialidad en la selva-fronteriza de Chiapas, México", $R e$ vista Mexicana de Ciencias Politicas y Sociales, 195, División de Estudios de Posgrado, Facultad de Ciencias Políticas y Sociales, UNAM, pp.163-194. 